\title{
Non-equilibrium dissociating nitrogen flow over a wedge
}

\author{
By D. J. KEWLEY AND H. G. HORNUNG \\ Department of Physics, Australian National University, Canberra
}

(Received 1 June 1973 and in revised form 2 February 1974)

\begin{abstract}
Experimental results for dissociating nitrogen flow over a wedge, obtained in a free-piston shock tunnel, are described. Interferograms of the flow show clearly the curvature of the shock wave and the rise in fringe shift after the shock associated with the dissociation. It is shown that the shock curvature at the tip of the wedge can be used to calculate the initial dissociation rate and that it is a more sensitive indication of the rate than can be obtained from fringe shift measurements under the prevailing experimental conditions. Because the freestream dissociation fraction can be adjusted in the shock tunnel, the dependence on atomic nitrogen concentration of the dissociation rate can be determined by the shock curvature method. A detailed calculation of the flow field by an inverse method, starting from the measured shock shape, shows good agreement with experiments.
\end{abstract}

\section{Introduction}

This study is concerned with hypersonic flow of nitrogen over a wedge in the case when the effects of chemical non-equilibrium behind the shock wave are significant. In such a situation the temperature rise across the shock requires the internal energy modes of the gas to be excited to a new equilibrium condition. The gas is able to adjust to this new condition only at a finite rate, so that a relaxation zone with a thickness typical of this rate and the flow speed follows the shock. The pressure and flow velocity are only relatively slightly affected by the relaxation process, so that the strong decrease in translational temperature associated with the redistribution of energy in the relaxing mode is accompanied by a strong rise in density. This in turn causes the streamtubes to contract across the relaxation zone, with the result that the shock curves towards the wedge.

Non-equilibrium wedge flows have been discussed thoroughly in a number of theoretical treatments (see Sedney 1961; Clarke 1969). They yield the important result that the shock curvature at the tip of the wedge is found to be proportional to the initial dissociation rate. The structure of the relaxation zone, which depends on the form of the relaxation rate, has also been calculated by the method of characteristics. Experimental results have been obtained in oxygen by Spurk \& Bartos (1966) and by Spurk (1970). The shock curvature method has been used experimentally by Smith \& Wegener of Yale University (in a study 
available only as an internal report) to measure evaporation rates in flow over a wedge. This work is also reported by Becker (1972, p. 187).

The aim of the present work is to make an experimental study of non-equilibrium dissociation in the flow of nitrogen over a wedge, using the free-piston shock tunnel at the Australian National University (see Stalker 1972) with a view to demonstrating the feasibility of obtaining the initial dissociation rate of nitrogen at high temperatures by measuring the curvature of the shock at the tip of the wedge. The most common method of measuring dissociation rates is to study the density rise after a normal shock in a shock tube. At high shock speeds, that is, at high temperatures, it is difficult to measure the initial rate accurately by this method, while the shock curvature technique can be expected to give good results at high temperatures. Another interesting feature of the curvature technique is that the free-stream degree of dissociation may be varied in a shock tunnel, so that, ideally, the relative efficiency of atomic and molecular nitrogen as a collision partner in dissociation can also be measured.

In addition, an inverse technique is to be used to make numerical computations of the flow, starting from the measured shock shape and integrating towards the body. The calculated body shape and the structure of the relaxation zone can then be compared with experimental results obtained by optical interferometry.

\section{Calculation of initial shock curvature}

The shock-wave curvature $\kappa$ at the tip of the wedge for any non-equilibrium flow can be written (see Sedney 1961; Becker 1972) as

$$
\kappa=\frac{\Sigma h_{i} W_{i}}{\rho u h_{\rho}} /\left\{\frac{\partial \xi}{\partial \beta} / \sin \lambda+\left(M_{f}^{2}-1\right) \frac{\partial p}{\partial \beta} / \rho u^{2} \cos \lambda\right\},
$$

where $h=h\left(p, \rho, c_{j}\right)$ is the specific enthalpy,

$$
h_{i}=\left(\partial h / \partial c_{i}\right)_{p, \rho, c_{j}(i \neq j)}, \quad h_{\rho}=(\partial h / \partial \rho)_{p, c_{j}} .
$$

$p, \rho, u$ and $T$ denote the pressure, density, velocity and translational temperature behind the shock, $\theta$ is the wedge inclination to the flow, $\beta$ is the shock inclination to the flow, $\xi$ is the streamline inclination behind the shock, $\lambda=\beta-\theta$ and $M_{f}$ is the frozen Mach number $u / a_{f}$, where $\alpha_{f}=\left[(\partial p / \partial \rho)_{s, c_{j}}\right]^{\frac{1}{2}}$ is the frozen speed of sound behind the shock, $s$ being the specific entropy. The reaction rate is

$$
D c_{i} / D t=W_{i}\left(p, \rho, c_{j}\right),
$$

where $\left\{c_{j}\right\}$ is a finite set of relaxing parameters, which may be species concentrations of energies of internal modes (see Hayes \& Probstein 1965, p. 537).

If dissociation does not occur until the vibrational mode is fully excited and in equilibrium, then, for an inviscid partially dissociated nitrogen free stream,

and

$$
\begin{gathered}
\frac{\Sigma h_{i} W_{i}}{\rho u h_{\rho}}=\frac{D \alpha}{D t} \frac{4 p\left|\rho(1+\alpha)-R \Theta_{d}(1+\alpha)\right| \mathscr{M}}{u a_{f}^{2}(3 \cdot 5-0 \cdot 5 \alpha)}, \\
p=\rho R T(1+\alpha) / \mathscr{M}
\end{gathered}
$$


where $\Theta_{d}$ is the characteristic dissociation temperature of nitrogen $\left(=113200^{\circ} \mathrm{K}\right)$, $\alpha$ is the mass fraction of atomic nitrogen in the free stream, $R$ is the universal gas constant and $\mathscr{M}$ is the molecular weight of nitrogen.

The conditions immediately behind the shock (subscript $s$ ) now have to be obtained from the free-stream conditions (subscript $\infty$ ) and the shock jump relations

$$
\begin{gathered}
p_{s}=p_{\infty}+\rho_{\infty} u_{\infty}^{2} \sin ^{2} \beta(1-\epsilon), \\
h_{s}=h_{\infty}+\frac{1}{2} u_{\infty}^{2} \sin ^{2} \beta\left(1-\epsilon^{2}\right),
\end{gathered}
$$

where $\epsilon \rho_{s}=\rho_{\infty}$. The density can also be expressed in terms of pressure, enthalpy and $\alpha$ by using (3) and (4):

$$
\rho_{s}=(4 \cdot 5+0 \cdot 5 \alpha) p_{s} /\left\{\left(h_{s}-\alpha R \Theta_{d} / \mathscr{M}\right)(1+\alpha)\right\}
$$

If the test flow is generated by a nozzle expansion, the vibrational energy may be frozen at a temperature $T_{v}$ greater than the free-stream translational temperature. Thus $h_{\infty}$ in (6) would be of the form

$$
h_{\infty}=(3 \cdot 5+1 \cdot 5 \alpha) p_{\infty} / \rho_{\infty}(1+\alpha)+\alpha R \Theta_{d} / \mathscr{M}+R \Theta_{v}(1-\alpha) / \mathscr{M}\left\{\exp \left(\Theta_{v} / T_{v}\right)-1\right\},
$$

where $\Theta_{v}$ is the characteristic temperature for vibration. Thus $p_{s}, \rho_{s}$ and $h_{s}$ can be found for a given shock angle and free stream by solving (5)-(8) iteratively, starting with $\epsilon=0$. A solution can also be obtained at the tip of the wedge if only the wedge angle and free-stream conditions are known. For a straight shock,

and thus,

$$
\tan (\beta-\theta)=\epsilon \tan \beta,
$$

$\tan \beta=\epsilon^{-\frac{1}{2}} f\left(\eta_{w}\right)$

where $f(\eta)=2 \eta /\left[1+\left(1-4 \eta^{2}\right)^{\frac{1}{2}}\right]$ and $\eta_{w}=\epsilon^{\frac{1}{2}} \tan \theta /(1-\epsilon)$ (see Hayes \& Probstein 1965, p. 218). Starting with a guessed $\epsilon$, continued iteration converges to the correct $\beta$ at the tip of the wedge.

Provided that the rate constants are known, the conditions behind the shock determine the initial dissociation rate, which is assumed to be given by the linear rate law (see Vincenti \& Kruger 1965, p. 232):

$$
D \alpha / D t=2 \rho(1-\alpha)\left\{k_{f 1} \alpha+\frac{1}{2} k_{f 2}(1-\alpha)\right\} / \mathscr{M},
$$

where recombination is neglected. Equation (10) combines the two reactions possible because of the partially dissociated free stream:

$$
\left.\begin{array}{l}
\mathrm{N}_{2}+\mathrm{N} \stackrel{k_{f f_{3}}}{\rightleftharpoons} 2 \mathrm{~N}+\mathrm{N}, \\
\mathrm{N}_{2}+\mathrm{N}_{2} \stackrel{k_{f 2}}{\rightleftharpoons} 2 \mathrm{~N}+\mathrm{N}_{2} .
\end{array}\right\}
$$

In the relevant temperature range $\left(9000-16000^{\circ} \mathrm{K}\right)$ two experimental evaluations of nitrogen dissociation rates have been made: Appleton, Steinberg \& Liquornik (1968) give

$$
\left.\begin{array}{l}
k_{f 1}=(1 \cdot 6 \pm 0 \cdot 6) \times 10^{22} T^{-1 \cdot 6} \exp \left(-\Theta_{d} / T\right) \mathrm{cm}^{3} \mathrm{~mole}^{-1} \mathrm{~s}^{-1}, \\
k_{f 2}=(3 \cdot 7 \pm 0 \cdot 9) \times 10^{21} T^{-1 \cdot 6} \exp \left(-\Theta_{d} / T\right) \mathrm{cm}^{3} \mathrm{~mole}^{-1} \mathrm{~s}^{-1},
\end{array}\right\}
$$




\begin{tabular}{|c|c|c|c|c|c|}
\hline Figure numb & ber & $2(a)$ & $2(b)$ & $2(c)$ & $2(d)$ \\
\hline Reservoir & $\left\{\begin{array}{l}h\left(\mathrm{~cm}^{2} \mathrm{~s}^{-2}\right) \\
T\left({ }^{\circ} \mathrm{K}\right) \\
p\left(\mathrm{dyn} \mathrm{cm}^{-2}\right)\end{array}\right.$ & $\begin{array}{l}2 \cdot 34 \times 10^{11} \\
9380 \\
2 \cdot 35 \times 10^{8}\end{array}$ & $\begin{array}{l}3.0 \times 10^{11} \\
10000 \\
2 \cdot 31 \times 10^{8}\end{array}$ & $\begin{array}{l}3.4 \times 10^{11} \\
10400 \\
2 \cdot 28 \times 10^{8}\end{array}$ & $\begin{array}{l}4.0 \times 10^{11} \\
10900 \\
2 \cdot 27 \times 10^{8}\end{array}$ \\
\hline $\begin{array}{l}\text { Free } \\
\text { stream }\end{array}$ & $\left\{\begin{array}{l}\alpha \\
\rho\left(\mathrm{g} \mathrm{cm}^{-3}\right) \\
U\left(\mathrm{~km} \mathrm{~s}^{-1}\right) \\
T\left({ }^{\circ} \mathrm{K}\right) \\
p\left(\mathrm{dyn} \mathrm{cm}^{-2}\right) \\
M\end{array}\right.$ & $\begin{array}{l}0 \cdot 12 \\
4 \cdot 6 \times 10^{-6} \\
5 \cdot 8 \\
1530 \\
2 \cdot 4 \times 10^{4} \\
6 \cdot 8\end{array}$ & $\begin{array}{l}0 \cdot 20 \\
3 \cdot 9 \times 10^{-6} \\
6 \cdot 3 \\
1730 \\
2 \cdot 4 \times 10^{4} \\
6 \cdot 7\end{array}$ & $\begin{array}{l}0 \cdot 26 \\
3 \cdot 5 \times 10^{-6} \\
6 \cdot 7 \\
1810 \\
2 \cdot 4 \times 10^{4} \\
6 \cdot 7\end{array}$ & $\begin{array}{l}0 \cdot 35 \\
3 \cdot 0 \times 10^{-6} \\
7 \cdot 1 \\
1900 \\
2 \cdot 3 \times 10^{4} \\
6 \cdot 7\end{array}$ \\
\hline
\end{tabular}

TABLE 1. Reservoir and free-stream conditions

determined in shock-tube experiments with an argon heat bath $\left(8000-15000{ }^{\circ} \mathrm{K}\right)$; Hanson \& Baganoff (1972) give

$$
\left.\begin{array}{l}
k_{f 1}=2 \cdot 2 \times 10^{26 \pm 0.3} T^{-2.5} \exp \left(-\Theta_{d} / T\right) \mathrm{cm}^{3} \mathrm{~mole}^{-1} \mathrm{~s}^{-1}, \\
k_{f 2}=3.9 \times 10^{33 \pm 0.3} T^{-4.5} \exp \left(-\Theta_{d} / T\right) \mathrm{cm}^{3} \mathrm{~mole}^{-1} \mathrm{~s}^{-1},
\end{array}\right\}
$$

determined from the pressure history at the closed end of a shock tube with undiluted nitrogen $\left(5700-12000^{\circ} \mathrm{K}\right)$. Equations (12) and (13) are adopted here for comparison with experimental results.

The shock curvature at the tip of the wedge may now be calculated by substituting the conditions just behind the shock and the dissociation rate into (1). To determine the variation of shock curvature with distance from the tip one would have to use the method of characteristics, which is beyond the scope of this work.

\section{Experiment}

\subsection{Equipment and experimental conditions}

A sharp flat plate $15 \mathrm{~cm}$ wide and $14 \mathrm{~cm}$ long with adjustable incidence is used as a model in the A.N.U. free-piston shock tunnel known as T 3, with a conical nozzle of included angle $15^{\circ}$ and exit diameter $20.3 \mathrm{~cm}$. The flow is studied with a Mach-Zehnder interferometer, using an exploding-wire light source.

The wedge angle, that is, the plate incidence $\theta$, is varied from $35^{\circ}$, giving a nearfrozen flow with a straight shock, to $43^{\circ}$, which is typically near the detachment limit. Throughout this range the shock curvature increases rapidly. The reservoir specific enthalpy $h_{0}$ is varied from $2 \times 10^{11}$ to $4 \times 10^{11} \mathrm{~cm}^{2} \mathrm{~s}^{-2}$ with corresponding reservoir temperatures $T_{0}$ ranging from 9300 to $11000^{\circ} \mathrm{K}$. The conditions in the free stream are calculated numerically using the non-equilibrium nozzle-flow program of Lordi, Mates \& Moselle (1966) with a modification taking account of vibrational relaxation (without vibration-dissociation coupling; see table 1).

\subsection{Results with $\theta=35^{\circ}$}

Throughout the enthalpy range, a wedge at $35^{\circ}$ incidence produces a virtually straight shock wave with a small curvature at the tip for the higher enthalpy case (see figure 1, plate 1). For a straight shock, $\beta$ and $\theta$ determine the density ratio 
$\rho_{s} / \rho_{\infty}$. From the fringe shift across the shock the density difference $\rho_{s}-\rho_{\infty}$ can be found. Thus, both densities can be determined from an interferogram such as that in figure 1. The error in $\rho_{\infty}$ from this determination is $\pm 6 \%$. This result can then be used with measurements of the free-stream Pitot pressure, which is approximated very closely by $\rho_{\infty} u_{\infty}^{2}$, to determine the free-stream speed to within $\pm 8 \%$. The density ratio $\rho_{s} / \rho_{\infty}$ is sensitive to $\alpha$ and $T_{v}$, the two non-equilibrium variables of the nozzle flow. The third variable needed to determine $\rho_{s} / \rho_{\infty}$ from (4)-(9) is $p_{\infty}$, which, with given reservoir conditions for a given nozzle, depends only on the relaxation processes in the nozzle, and these are characterized by $\alpha$ and $T_{v}$. Thus, the $35^{\circ}$ wedge results serve to confirm the calculations of free-stream conditions. The agreement between the nozzle-flow calculations and the measured results is within the estimated errors throughout the enthalpy range. The shot-to-shot repeatability of the free-stream conditions in the facility is also well within the estimated errors.

The theoretical shock angles on a $35^{\circ}$ wedge are found to be up to one degree smaller than the measured values. This is accounted for by the displacement effect of the boundary layer on the wedge. The boundary-layer thickness can be measured approximately from the interferogram, in which the density rise near the wall can be clearly seen as a positive fringe shift. The simple correction of adding $0 \cdot 5^{\circ}$ to $\theta$ for the displacement effect gives approximately the right shock angles throughout the enthalpy range.

\subsection{Shock curvature due to causes other than relaxation}

If shock curvature due to relaxation is to be measured, other causes contributing to shock curvature must be considered. One of these is the boundary-layer growth on the wedge. It modifies the term $\Sigma h_{i} W_{i}$ in (1) to

$$
\left.\Sigma h_{i} W_{i}-(d \xi / d s)\left\{\tan \lambda\left(M_{f}^{2}-1\right)-\cot \lambda\right)\right\} \rho u h_{\rho},
$$

where $d \xi / d s$ is the streamline curvature at the tip caused by boundary-layer growth. The boundary-layer thickness can be considered to grow as $s^{\frac{1}{2}}$ when the shock-boundary-layer interaction parameter, $\bar{x}=M_{j}^{2} / R e^{\frac{1}{2}}$, is less than one, where $R e$ is the Reynolds number based on conditions just behind the shock and on the length $s$. As $M_{f}$ is typically less than 2 and $R e$ based on $s=1 \mathrm{~cm}$ is about $5 \times 10^{3}$ behind the shock, $\bar{\chi}$ is typically less than 1 at $s>0.2 \mathrm{~mm}$. However, the simplest way of determining the boundary-layer contribution is to measure the shock curvature of the low-wedge-angle results $\left(\theta=35^{\circ}\right)$, where the boundary layer is the main cause of shock curvature. This leads to a value of about $0.01 \mathrm{~cm}^{-1}$ at $1 \mathrm{~mm}$ from the leading edge. This is a conservative estimate of the boundarylayer contribution at higher wedge angles, because the boundary-layer thickness and displacement effect decrease as the wedge angle is increased. It represents about $3 \%$ of the measured initial curvatures at higher angles.

Another contribution to the shock curvature is the effect of the conically divergent free stream. This effect is discussed in detail by Hall (1963), whose results were used to calculate this correction for each experimental case. The error incurred by neglecting the divergence effect in the present experimental cases always represents less than $8 \%$ of the measured initial curvature. The magnitude 

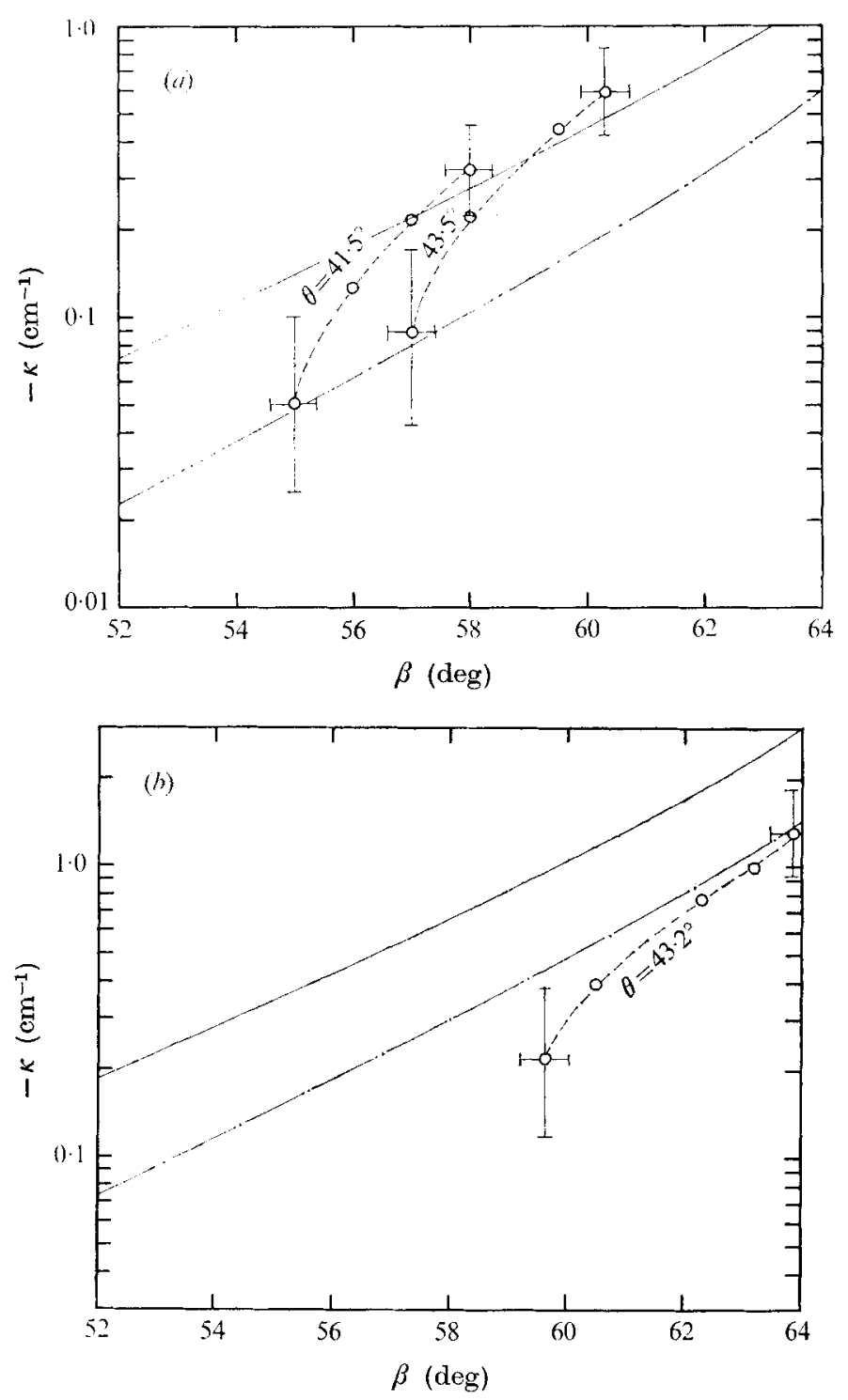

Figures $2(a, b)$. For legend see facing page.

of this eflect can be observed to be small by performing wedge-flow experiments with similar shock angles under frozen-flow conditions in the same nozzle, by lowering the enthalpy. In such experiments the shock curvature is observed to be so small that it is not possible to resolve its cause. Near the detachment limit a third contribution arises because the flow downstream of the shock is subsonic. Consequently, the expansion at the trailing edge of the wedge is able to influence the whole flow field up to the tip, thus curving the shock from the tip to a point opposite the trailing edge, where the flow will have reached sonic speed. The maximum change in shock angle possible owing to this contribution over the length of the wedge is $0.5^{\circ}$ at the free-stream Mach numbers of present interest. 

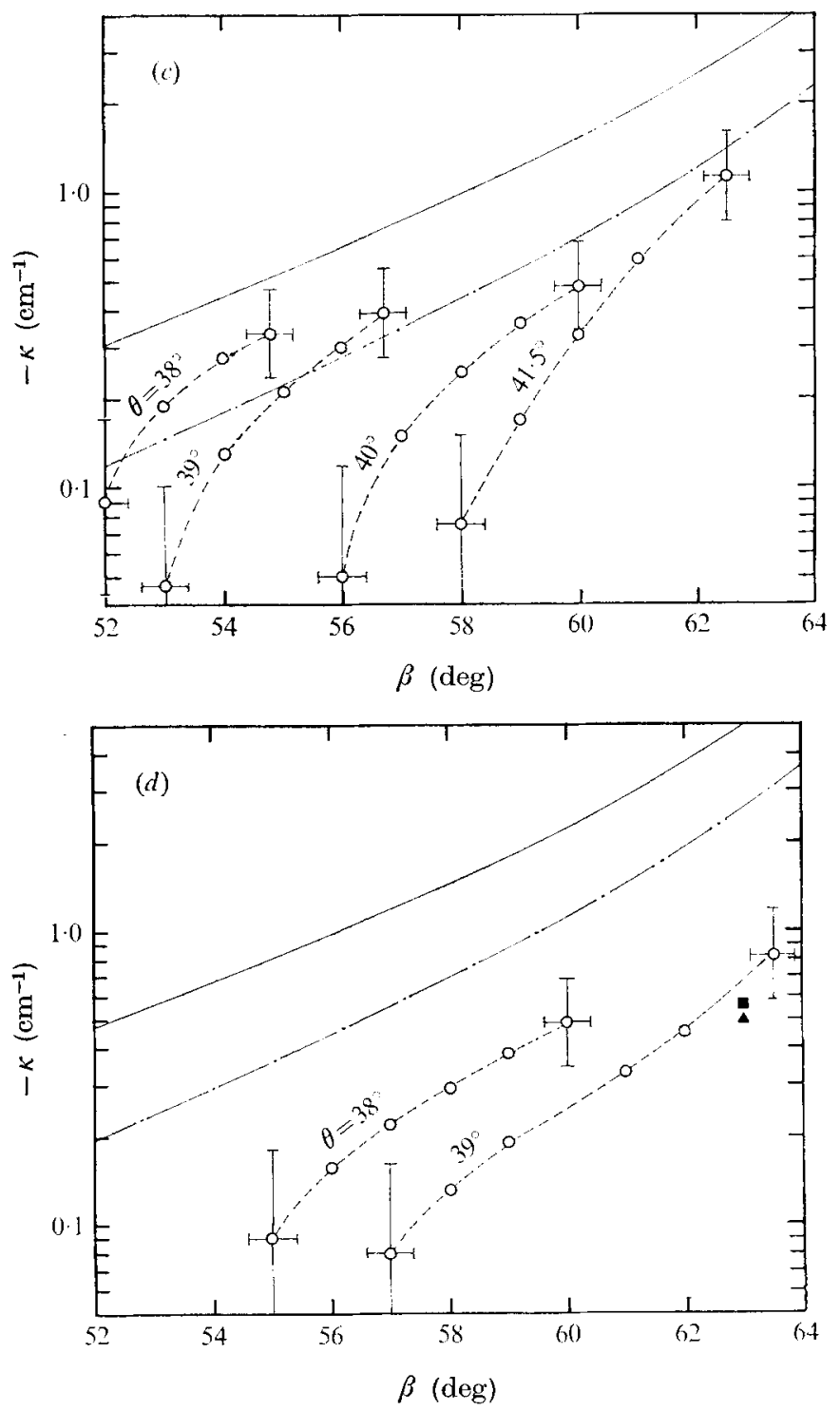

FIGURE 2. Comparison of experimental shock curvature and predicted initial shock curvature. - - O--, experimental; - - - predicted according to (12); - - predicted according to equation (13). Note that for agreement between experiment and theory, right-hand end of experimental curve should fall on theoretical curve. For reservoir and free-stream conditions for $(a)-(d)$ see table $1 . \mathbf{\square}$, equation (12), $k_{f 2}$ only; $\boldsymbol{\Lambda}$, equation (13), $k_{f 2}$ only.

\subsection{Experimental initial shock curvature}

The experimental shock shape is obtained from the interferograms by measuring, at both edges of each fringe, for the first 20 fringes, the distance from the wedge surface to the point where the fringe first registers the density rise at the shock front. These measurements are made to an accuracy of $\pm 0.04 \mathrm{~mm}$ in the region of interest, which is restricted to a distance of about $1 \mathrm{~cm}$ from the leading edge. 
A least-squares fit to these points then gives the shock shape as a polynomial in distance along the wedge surface, from which the shock-wave curvature $\kappa$ and angle $\beta$ may then be calculated as functions of distance along the wedge.

To compare the experimental results with theory, the initial curvature is calculated at each tunnel condition for a number of values of $\beta$. This is done using the free-stream conditions as calculated from the reservoir conditions with the rates for nitrogen recommended by Appleton et al. (1968) and with the vibrational relaxation rate recommended by Millikan \& White (1963). For the determination of the initial shock curvature from (1) the dissociation rates of Appleton et al. (1968), equation (12), and of Hanson \& Baganoff (1972), equation (13), are used, and the vibrational mode is assumed to be in equilibrium downstream of the shock. It should be pointed out at this stage that the nozzle flow is very insensitive to the recombination rate in comparison with the sensitivity of the shock curvature to the dissociation rate, and that the temperatures involved in the nozzle flow are considerably lower than those behind the wedge shock, so that the choice of rates does not affect the nozzle flow significantly, and the conditions (in the nozzle flow) are within a temperature range where the rates are relatively well known. Similarly, the effect of altering the vibrational relaxation rate in the nozzle-flow calculation from $\frac{1}{50}$ of Millikan \& White's (1963) rate to an infinite rate is to alter the initial shock curvature by no more than $\pm 12 \%$ in the present range of interest.

The results of the initial shock curvature calculations are plotted in figures $2(a)-(d)$, in order of increasing reservoir enthalpy, as graphs of initial curvature against shock angle for both the dissociation rates of Appleton et al. and of Hanson \& Baganoff. Superimposed on these figures are the curvature-slope traces of some experimental shock waves. It should be pointed out that each experimental curve represents the $\kappa(\beta)$ trace of the whole of the shock, so that the righthand end of the trace gives the values of $\kappa$ and $\beta$ at the tip of the wedge, whereas the calculated curves represent tip values only. Thus for agreement of experiment with predictions according to previously measured rates, the right-hand (large $\beta$ ) end of the experimental curve should fall on the calculated curve. The error bars indicate the estimated uncertainty in the experimental results from all sources.

At the lower reservoir enthalpy (figure $2 a$ ) the experimental initial shock curvature is seen to be in good agreement with the values predicted by the dissociation rates of Hanson \& Baganoff. At the intermediate reservoir enthalpies (figures $2 b, c$ ) the experimental curvature is in approximate agreement with the value predicted using the dissociation rates of Appleton et al. It can be seen from figure $2(c)$ that the experimental curvature at the tip of the wedge exhibits a trend with $\beta$ which is not quite as pronounced as that of either of the calculated curves. This means that in the present experiment the initial dissociation rate may be a slightly weaker function of temperature (which increases as $\beta$ is increased) than the rates measured by other experimenters. This is further confirmed by the decrease of experimental tip curvature relative to the calculated $\kappa$ as the reservoir enthalpy is increased, especially in view of the results presented in figure $2(d)$, which show that, at the highest reservoir enthalpy, the experimental tip curvature is well below the predicted curves. It should be remembered, 
however, that the rates (12) and (13), which apply up to $12000^{\circ} \mathrm{K}$ and $15000^{\circ} \mathrm{K}$ respectively, have to be extrapolated well beyond their range of applicability for figure $2(d)$, the temperature after the steeper shock being $16300^{\circ} \mathrm{K}$.

It is important to note that the present results represent dissociation rate measurements at a particular value of the dissociation fraction $\alpha$, which is known both from nozzle-flow calculations and non-reacting wedge-flow experiments at small $\beta$, and that the dissociation rate may well depend on $\alpha$ in a more complicated way than (10). Unlike previous measurements of the dissociation rate, the present method is therefore able to resolve the dependence of the total dissociation rate on $\alpha$ without relying on (10). Thus, the trend of $\kappa$ with reservoir enthalpy (and therefore with $\alpha$ ) might be interpreted as a failure of (10).

By considering the contribution to the curvature from $k_{f 2}$ only, the values of tip curvature predicted by both Appleton et al. and by Hanson \& Baganoff are found to agree with experimentally observed curvatures at the highest reservoir enthalpy and at $\beta=63^{\circ}\left(T_{s}=16300^{\circ} \mathrm{K}\right.$; see figure $\left.2 d\right)$. Possible reasons for this are that the rates may be much smaller than the accepted values, or that (10) may not be valid. The magnitude of the discrepancy indicates that the former reason is more likely. Care must be exercised in reaching a definite conclusion, however, because the temperature of this experiment is well above the limits of the ranges of applicability of (12) and (13).

To show the source of the experimental information, figure 3 (plate 2) shows an interferogram corresponding to the case $\theta=41.5^{\circ}$ of figure $2(a)$. The curvature of the shock as well as the rise in fringe shift between shock and body, corresponding to the density rise due to dissociation, are clearly visible. The rise in fringe shift close to the body indicates the boundary-layer thickness. The kinks in the fringes right on the body surface are due to an optical effect which is observed when the model is well aligned with the light beam.

\section{Calculation of the flow field from the measured shock}

Inverse techniques of calculating supersonic flow fields, in which the shock shape is assumed to be known and the body shape emerges as part of the solution, are open to criticism because of the question of whether to each shock shape there corresponds a single body shape. This objection disappears if the flow field downstream of the shock wave is supersonic throughout. Thus, an inverse method in which the flow field is calculated by starting from the measured shock-wave shape should give the correct body shape and fringe shift distribution, provided that the reaction rates are correct. Of three calculations made with different reaction rates at conditions corresponding to those of figure 3 , one is shown in figure 4 . The method used is basically that of Garr \& Marrone (1963) with modifications as described in Hornung (1972b). The reaction rates for this calculation are taken from best fits to shock-tube measurements made at A.N.U. by Kewley $\&$ Hornung (1974). These fall between the rates measured by Appleton et al. (1968) and by Hanson \& Baganoff (1972) and therefore predict curvatures lying between the two calculated curves of figure 2. As for the rates (12) and (13), however, conditions in figure $2(d)$ require extrapolation beyond the highest temperature 


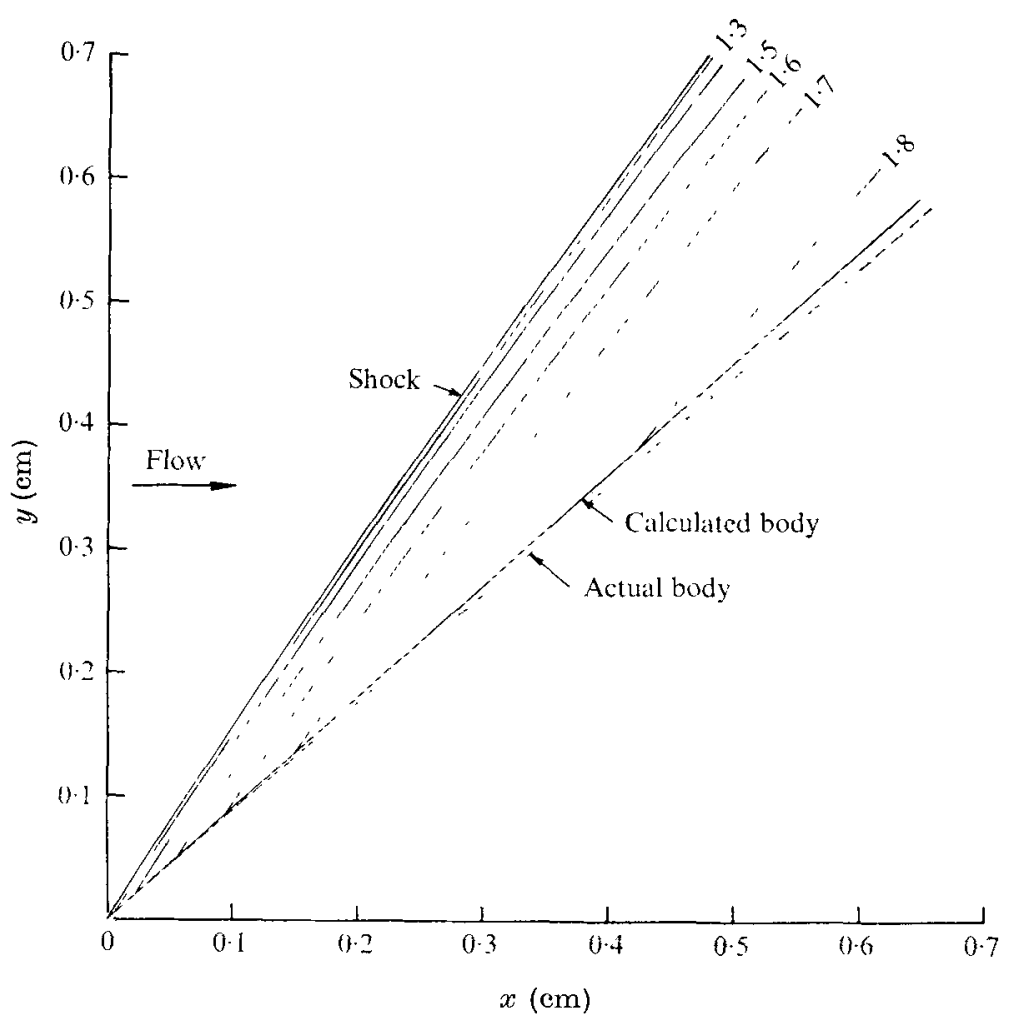

Figure 4. Flow field calculated from measured shock shape of figure 3 by inverse method, using reaction rates of Kewley \& Hornung (1974). Calculated body slope $=42 \cdot 2^{\circ}$. Actual plate incidence $=41 \cdot 5^{\circ}$. The curves represent contours of fringe shift, the value of which relative to the free stream is indicated by the numbers.

of the shock-tube measurements $\left(14000^{\circ} \mathrm{K}\right)$. An additional refinement of this calculation is that vibrational relaxation was accounted for by a Landau-Teller model with Millikan \& White's (1963) rates. Figure 4 gives the calculated fringe shift contours in the region close to the tip. It can be seen that the calculated body shape is indistinguishable from a straight wedge, but the calculated $\theta$ is $0.7^{\circ}$ larger than the actual wedge angle. This is to be compared to the approximate correction of $0.5^{\circ}$ for the boundary-layer displacement effect applied in \$3.2. The predicted body points are also shown in figure 5 as a terminator on each curve. The actual body position is indicated by a cross-hatehed bar. It should be pointed out that the induction time phenomenon which was observed previously (Hornung $1972 a$ ) is a small effect here.

A fringe shift profile across the shock layer and normal to the shock, taken at $0.6 \mathrm{~cm}$ from the tip of the wedge, is shown in figure 5 . The profile is chosen to be close to the tip to minimize the distortion of the fringe due to three-dimensional effects. Here the fringe shift according to all three sets of reaction rates is compared with experiment. It is immediately clear that the experimental results are quite unable to resolve the difference in fringe shift predicted by the three rates. Contrast this with figure $2(a)$, where the difference between the rates of 


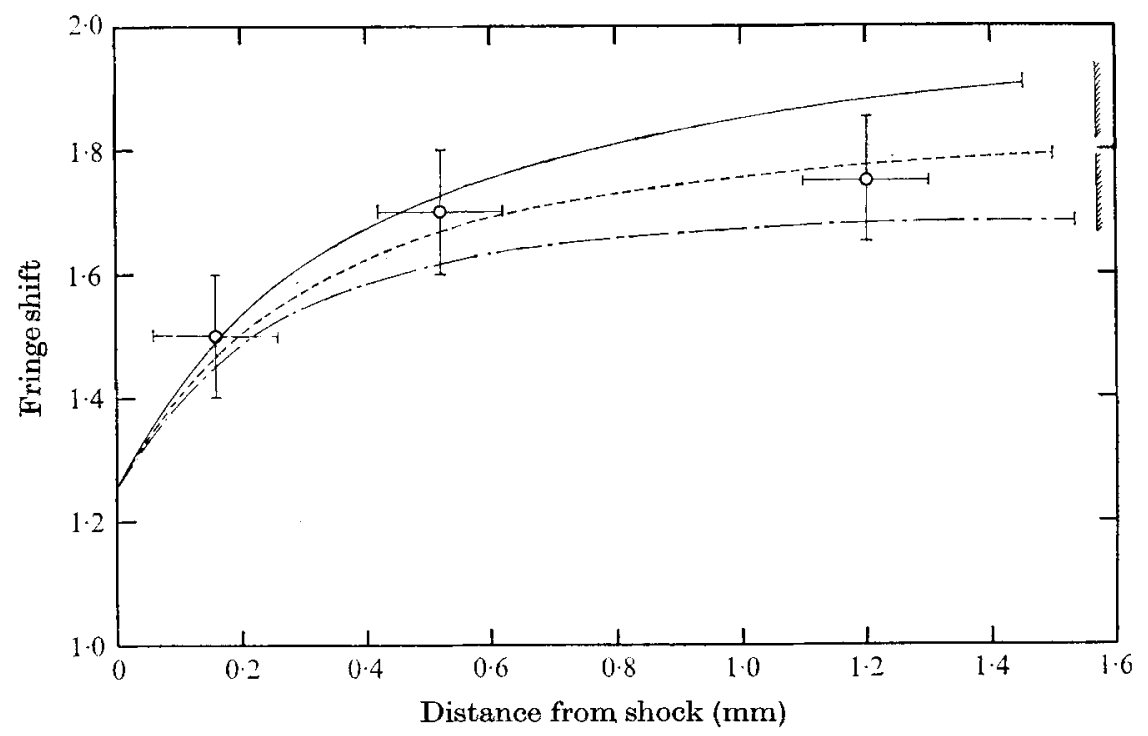

Figure 5. Profile of fringe shift across the shock layer at $0.6 \mathrm{~cm}$ from the tip of the wedge at conditions corresponding to figure 3 . $x_{-}$, experiment; _- - - calculated with (12); calculated with (13); - - --, calculated with rates of Kewley \& Hornung (1974). The curves are terminated at the predicted body point. $I I I I I I I I I$, indicates the actual body position.

Appleton et al. and Hanson \& Baganoff amounts to a factor of about 2.5 and the error bars of the experiment clearly favour the rates of Hanson \& Baganoff under these conditions. While one must be careful to remember that the shock curvature measures the initial rate only, it is clear that it doos this more accurately than the fringe shift measures the overall rate, under the present conditions.

\section{Conclusions}

The influence of relaxation effects due to dissociation on hypersonic wedge flows of nitrogen is studied experimentally. The curved shock wave and relaxation zone characterized by a rise of density can be seen clearly in interferograms of the flow. The shock curvature at the tip of the wedge is a more sensitive indication of the dissociation rate than interferometric measurements of the density rise after the shock under the conditions of the present experiments. The shock curvature method can be used to measure the relative efficiency of molecular and atomic nitrogen as collision partners in dissociation because the frec-stream degree of dissociation in the shock tunnel can be adjusted. The indications of the present study are that the variation of the initial dissociation rate after an oblique shock increases slightly more slowly with temperature than predicted by previous measurements, and that the free-stream degree of dissociation affects the rate more strongly than expected.

The financial support of the facility by the Australian Research Grants Committee is gratefully acknowledged. We should also like to thank R. French and K. Smith for technical and photographic assistance. 


\section{REFERENCES}

Appleton, J. P., Steinberg, M. \& Lrquornik, D. J. 1968 J. Chem. Phys. 48, 599. BECKer, E. 1972 Ann. Rev. Fluid Mech. 4, 155.

CLARKE, J. F. 1969 Gasdynamics, vol. 1. Nonequilibrium Flous, part 1 (ed. P. P. Wegener). Dekker.

GarR, L. J. \& MARRone, P. V. 1963 Cornell Aeron. Lab. Rep. QM-1626-A-12 (II).

Hari, J. G. 1963 Cornell Aeron. Lab. Rep. no. 128.

Hanson, R. K. \& Baganoff, D. 1972 A.I.A.A.J. 10, 211.

Hayes, W. D. \& Probstern, R. F. 1965 Hypersonic Flow Theory, vol. 1. Academic.

Hornung, H. G. 1972 a J. Chem. Phys. 56, 3172.

Hornung, H. G. $1972 b$ J. Fluid Mech. 53, 149.

Kewley, D. J. \& Hornung, H. G. 1974 Chem. Phys. Lett. to appear.

Lordi, J. A., Mates, R. E. \& Moselle, J. R. 1966 N.A.S.A. Contr. Rep. CR-472.

Millikan, R. C. \& White, D. R. 1963 J. Chem. Phys. 39, 98.

SEDNEY, R. 1961 J. Aerospace Sci. 28, 189.

SPURK, J. H. 1970 A.I.A.A.J.8, 1039.

Spurk, J. H. \& Bartos, J. M. 1966 Phys. Fluids, 9, 1278.

Stalker, R. J. 1972 Aeron. J. 76, 374.

Vincenti, W. G. \& KrugeR, C. H. 1965 Introduction to Physical Gasdynamics. Wiley. 


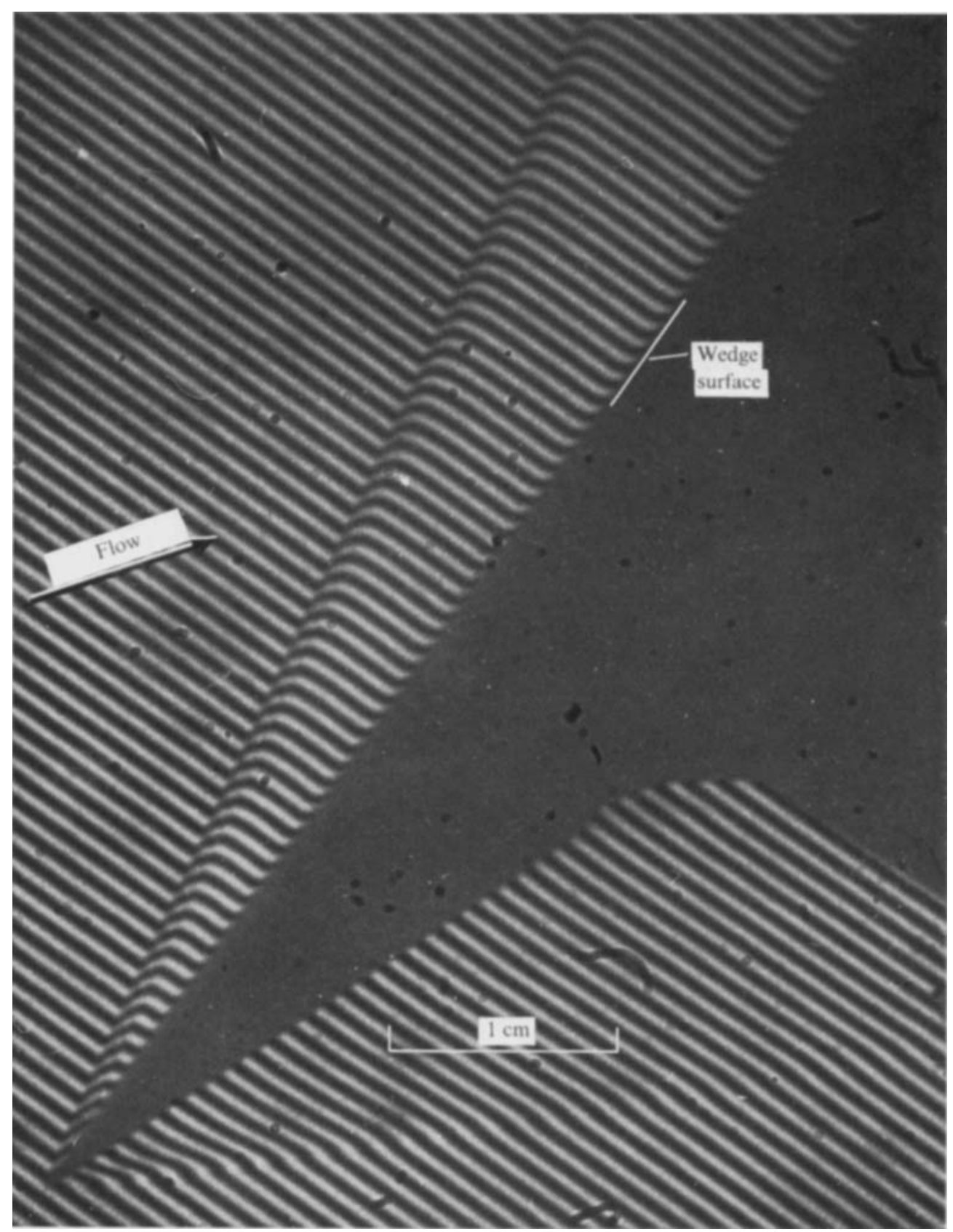

Figure 1. Interferogram of flow over flat plate at $35^{\circ}$ incidence. Conditions as in figure $2(a)$. 


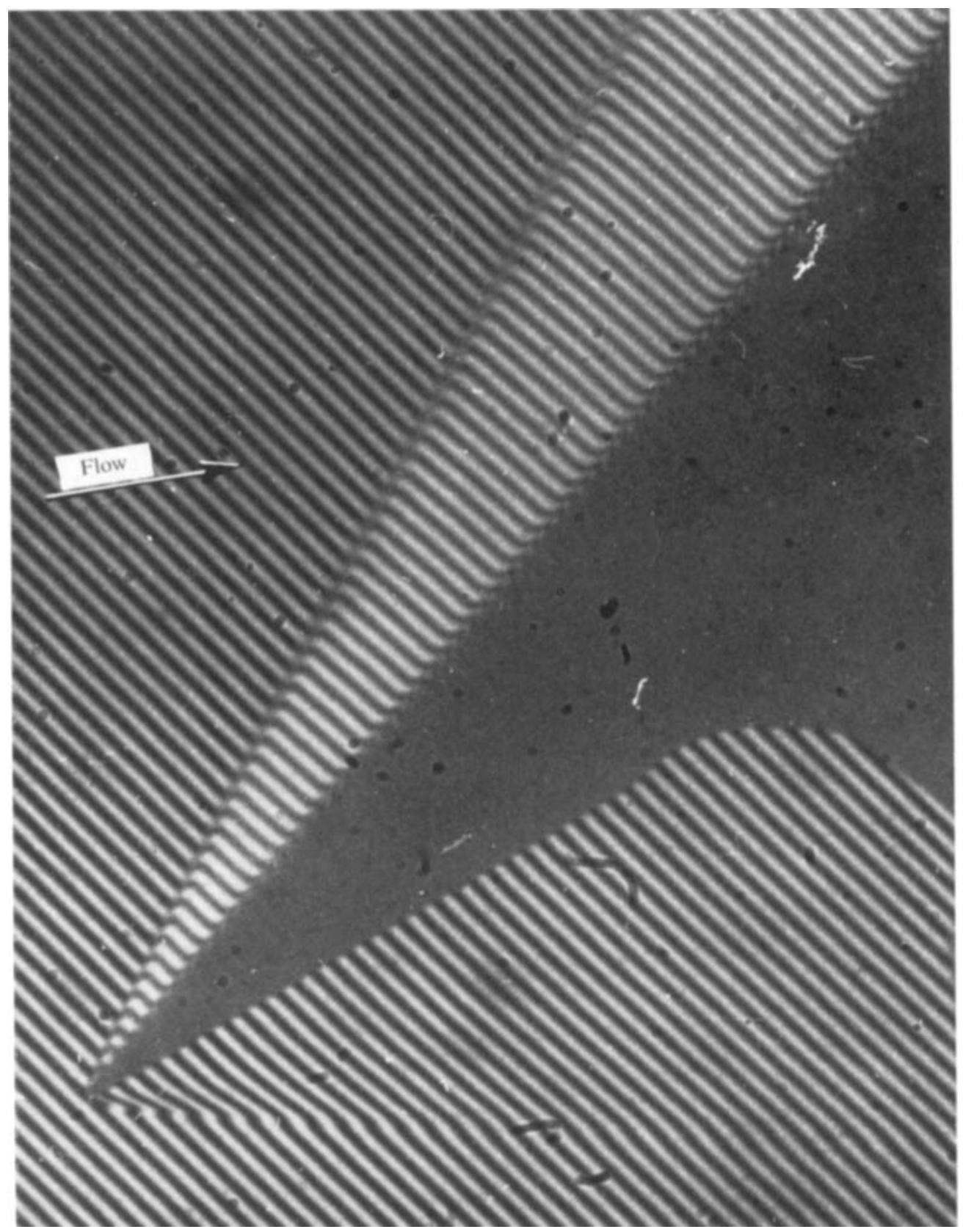

Figure 3. Interferogram of flow over a flat plate at $41 \cdot 5^{\circ}$ incidence. Conditions as in figure $2(a)$. 